\title{
Inhibition of Osteoarthritis in Rats by Electroporation with Interleukin-1 Receptor Antagonist
}

\author{
Zhen Sun1, Heyong Yin'1, Xiaoming Yu1, Xun Sun1, Bo Xiao1, Yichi Xu1 ${ }^{1}$, Zhiguo Yuan'1, \\ Haoye Meng1, Jiang Peng1*, Changlong Yu' ${ }^{2 *}$, Yu Wang1, Quanyi Guo', Aiyuan Wang1, \\ Shibi Lu1 \\ ${ }^{1}$ Beijing Key Lab of Regenerative Medicine in Orthopedics, Key Lab of Musculoskeletal Trauma \& War Injuries, \\ General Hospital of People's Liberation Army, Beijing, China \\ ${ }^{2}$ Institute of Sport Medicine, Peking University Third Hospital, Beijing, China \\ Email: *pengjiang301@126.com, *ycl123@vip.sina.com
}

Received 16 March 2016; accepted 12 June 2016; published 15 June 2016

Copyright (C) 2016 by authors and Scientific Research Publishing Inc.

This work is licensed under the Creative Commons Attribution International License (CC BY).

http://creativecommons.org/licenses/by/4.0/

(c) () Open Access

\begin{abstract}
Gene therapy constitutes a promising strategy for the treatment of osteoarthritis (OA). We assessed the use of electroporation (EP) of non-viral gene vectors, and compared its efficacy with that of adeno-associated virus (AAV) vectors. EP- and AAV-mediated delivery of human interleukin-1 receptor antagonist (hIL-1Ra) was localized performed in the joints of rats following induction of OA. mRNA levels for hIL-1Ra, IL-1 $\beta$, TNF- $\alpha$, MMP-13 and ADAMTS-4 in the cartilage and synovial tissues were analyzed. Structural analyses of the subchondral bone at the medial femoral condyle were performed by Micro-CT after treatment. Knee joint specimens were staining with hematoxylin and eosin and Saffron 0. Induction of hIL-1Ra by both EP and AAV inhibited inflammatory-induced subchondral bone reconstruction, and effectively suppressed IL-1 $\beta$ activity, as evidenced by decreased expression of MMP-13 and ADAMTS-4. Histological analyses revealed significant protection of cartilage, proteoglycan by EP and AAV. hIL-1Ra expression was similar in both the EP and AAV groups. Notably, this gene is not easier degraded transduced by EP compared with AAV. Taken together, these results show that EP offers transfection efficiency comparable to that of AAV, with the potential for longer gene expression, making EP a promising candidate for efficient non-viral delivery of $\mathrm{OA}$ gene therapy.
\end{abstract}

\section{Keywords}

Electroporation, Interleukin-1 Receptor Antagonist, Adeno-Associated Virus, Gene Therapy, Osteoarthritis, Cartilage, Synovium

\footnotetext{
*Corresponding authors.
}

How to cite this paper: Sun, Z., Yin, H.Y., Yu, X.M., Sun, X., Xiao, B., Xu, Y.C., Yuan, Z.G., Meng, H.Y., Peng, J., Yu, C.L., Wang, Y., Guo, Q.Y., Wang, A.Y. and Lu, S.B. (2016) Inhibition of Osteoarthritis in Rats by Electroporation with Interleukin-1 Receptor Antagonist. J. Biomedical Science and Engineering, 9, 323-336. http://dx.doi.org/10.4236/jbise.2016.97027 


\section{Introduction}

Osteoarthritis (OA) is the one of the most common joint diseases, characterized by loss of articular cartilage in combination with underlying bone changes, including sclerosis, bone cysts, subchondral bone sclerosis, and osteophyte formation [1] [2]. Due to the complex pathogenesis of this disease, there remain no satisfactory treatments for OA. Gene therapy is considered a promising new method for the treatment of joint-disorders such as OA, with the possibility of treating disease symptoms by targeting specific pathological mechanisms underlying pathogenesis [3] [4].

As a biological alternative for arthritis-based therapies, many studies have used gene vectors to treat arthritis in various animal models [5]-[7]. Alternative approaches for delivering gene products to the articular cartilage have also been used, with viral vectors being the most common method [8]-[11]. While most gene therapy approaches to date have employed viral vectors due to their high transfection efficiency in clinical trials, their potential for fatal defects may prevent any further clinical use, due to the potential for infectivity, oncogenic effects, immunogenicity, and other unexpected complications [7]-[14]. On the other hand, a number of studies examining the use of non-viral gene delivery systems have been reported, which may avoid many of these problems, including easier synthesis and modification, lower immunogenicity, and reduced safety risks [15]. As a non-viral vector, chitosan (CS) is considered to be an ideal candidate due to its superior biodegradability and biocompatibility profiles, and lower overall toxicity [16] [17]. However, its low transfection efficiency has limited further application as a non-viral gene delivery vector [18].

Designing an efficient protocol for OA treatment using non-viral gene delivery demands a number of considerations, including both the gene of interest for OA therapy, and the method for gene-delivery, with a need to balance both safety and efficacy. Interleukin-1 (IL-1) is the major cytokine involved in the pathology of OA [19] [20]. IL-1 has been shown to promote the synthesis of matrix metalloprotease, inhibit production of extracellular matrix proteins, and activate Rac1, resulting in up-regulation of ADAMTS-4, COLX, and Runx2 expression [21]. Furthermore, IL-1 is also known to induce the expression of other pro-inflammatory cytokines, such as, TNF- $\alpha$ and IL-6 [22] [23]. Suppression of these signals is known to occur through a variety of mechanisms, including expression of interleukin-1 receptor antagonist (IL-1Ra), a specific inhibitor that binds to the interleukin-1 receptor (IL-1R), but does not elicit an intracellular response [24]. The potential anti-arthritic effects of IL-1Ra have been reported in various animal models [8] [25]-[27]; however, effective delivery of IL-1Ra remains an issue.

Recently, electroporation (EP) has been identified as a safe and effective non-viral gene delivery method both in vitro and in vivo [28] [29]. DNA electroporation, also known as electrical field-mediated gene transfer, is able to transfer gene products across the cell membrane through use of short-voltage pulses [30]. EP has been shown to induce not only high levels of transgene expression but also sustained levels of protein gene production in transfected tissue. Preliminary results have shown efficacy in the treatment for rheumatoid arthritis, though similar approaches have not been done in OA [31]. EP-mediated delivery of gene products directly to the intraarticular tissue may provide an effective treatment for OA, while limiting systemic exposure through the blood stream. Here, we assessed the anti-arthritic effects of IL-1Ra gene transfection by EP in an OA rat model, and compared these effects with that of an adeno-associated virus (AAV) delivery system.

\section{Materials and Methods}

\subsection{Animal OA Model}

Animal studies were performed using 8-week old Male Sprague Dawley (SD) rats, weight 220 - 250 g, which were provided by the Experimental Animal Center of Chinese People’s Liberation Army (PLA) General Hospital. All protocols were approved by the Institutional Animal Care and Use Committee of the Chinese PLA General Hospital. The animals were randomized into treatment protocol groups. OA was induced by surgery of medial meniscus resection and anterior cruciate ligament transection, 1 week later, experimental OA pathology gradually developed over the surgery. Electroporation and AAV transfection of hIL-1Ra was initiated 1 week after the operation. Following treatment, knee joints were isolated once a week for the course of the study, and analyzed using histological, morphological, radiological, and molecular analyses. It is worth noting, in Results part, the 1-week time point represent 1 week after gene transfer not means 1 week after OA induced surgery. 


\subsection{Grouping}

Rats were randomly divided into 5 groups. Group 1: untreated, non-arthritic rats (normal group, $n=24$ ). Group 2: untreated arthritic rats that underwent OA surgery, with no follow up treatment (OA group, $n=24)$. Group 3 : OA rats treated with the hIL-1Ra/pCI-neo plasmid without transfection (naked plasmid (NP) group, $n=24$ ). Group 4: OA rats electroporated with the hIL-1Ra/pCI-neo plasmid (electroporation (EP) group, $n=24$ ). Group 5: OA rats treated with an adeno-associated virus (AAV) carrying the hIL-1Ra/pCI-neo plasmid (AAV group, $\mathrm{n}=$ 24). For groups 3 and 4, the dose of hIL-1Ra plasmid was acquired by Result 3.1.

\subsection{In Vivo Bioluminescence Imaging (BLI)}

Luciferase (Luc) was used as a reporter gene for bioluminescence observation. A luciferase plasmid was purchased from Genomeditech Co. (GM-0210PC) and injected into both sides of the rat knee joint through the patellar tendon. In vivo electroporation of the right joint was then used for transfection of this gene into cells.

To obtain bioluminescence images, rats were anesthetized using a nose cone delivering $2 \%$ isoflurane in $100 \%$ $\mathrm{O}_{2}$. D-Luciferin (40902ES03) was diluted to $30 \mathrm{mg} / \mathrm{mL}$ in Dulbecco's phosphate buffered saline (60147ES76), and $100 \mu \mathrm{L}$ D-Luciferin was directly administrated into both joints $1,3,7,14,21$, and 28 days post-electroporation. Next, the rats were placed in a light-obstructing chamber for $10 \mathrm{~min}$, and luciferase kinetics was recorded by consecutive 5 min acquisitions. A number of highlighted pixels were then counted using the instrument's imaging unit software to quantify the bioluminescent signal in the area shaped around each site of photon emission.

\subsection{Preparation of Human IL-1Ra Plasmid}

Human IL-1Ra cDNA was generously provided by Prof. Yu CL (Institution of Sports Medicine, Peking University Third Hospital, China). Full-length human IL-1Ra was cut from pcDNA3.1 (Invitrogen, USA). The human IL-1Ra plasmid was used in the treatment of OA, as described previously.

\subsection{In Vivo Electroporation and Viral Transduction with hIL-1Ra into Rat Joints}

Rats in groups 2 - 5 were anesthetized using a nose cone delivering $2 \%$ isoflurane in $100 \% \mathrm{O}_{2}$. For groups 3, 4, a mixture of hIL-1Ra plasmid in $100 \mu \mathrm{L}$ phosphate-buffered saline (PBS) was injected into the right knee joint using a syringe equipped with a 30-gauge needle; group 2 was treated with $100 \mu \mathrm{L}$ PBS. Following injection, the knee joint was flexed and extended several times. A pair of $8 \mathrm{~mm}$ electrode pads was then placed on the joint in group 4, as show in Figure 1(a). Electroporation was performed using an in vivo gene transfer instrument (Teresa Healthcare Sci-Twch Co., Ltd., China). This instrument can generate square wave signals with a pulse duration range of $1 \mathrm{~ms}-900 \mathrm{~ms}$.

For group 5, human IL-1Ra was also packaged into AAV vectors, the serotype is type 2, which were purified and titered as described previously. After packeaged, the delivery of AAV vectors, $2 \times 10^{9}$ vector genomes were diluted into $100 \mu \mathrm{L}$ solution, were directly injected into articular cavity, to achieve transfection in vivo. Not using AAV vectors transfect special cells, such as synoviocytes or chondrocytes or stem cells, in vitro, and then inject those cells into articular cavity.

\subsection{Real-Time PCR}

Following isolation of the knee joints, the articular cartilage and the synovium were separated, as described previously. Isolated tissues were then immersed in liquid nitrogen immediately in order to minimize RNA degradation. Total RNA was extracted in $1 \mathrm{~mL}$ TRIzol solution (Invitrogen, USA) for $24 \mathrm{~h}$ at $4^{\circ} \mathrm{C}$. cDNA was generated using the ReverTra Ace qPCR RT Kit (Toyobo, Japan) at $37^{\circ} \mathrm{C}$ for $15 \mathrm{~min}$, and at $98^{\circ} \mathrm{C}$ for $5 \mathrm{~min}$. Real-time PCR was performed using the SYBR Green Realtime PCR Master Mix-Plus (Toyobo). For each reaction, $1 \mu \mathrm{L}$ cDNA and $1 \mu \mathrm{L}$ of each primer were used. The primer sequences used are as follows: rat GAPDH forward primer 5'-ATGGTGAAGGTCGGTGTGAACG-3' and reverse primer 5'-TTACTCCTTGGAGGCC ATGTAG-3'; hIL-1Ra forward primer 5'-AGATGTGCCTGTCCTGTGTC-3' and reverse primer 5'-CCTGC TTTCTGTTCTCGCTC-3'; rat IL-1 $\beta$ forward primer 5'-TTGTGGCTGTGGAGAAGCTG-3' and reverse primer 5'-GCCGTCTTTCATACACAGG-3'; rat TNF- $\alpha$ forward primer 5'-CATGATCCGAGATGTGGAACT 
(a)

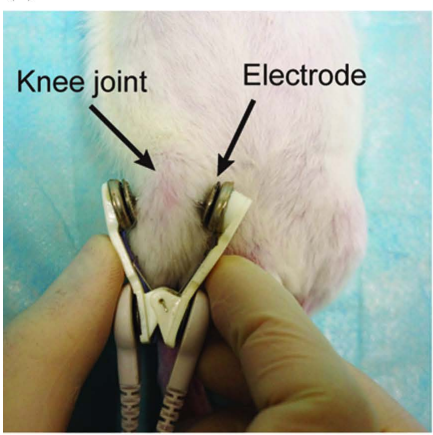

(b)

\begin{tabular}{|c|c|c|}
\hline Group & Pulse width (ms) & Plasmid dose (ug) \\
\hline Group a & 10 & 50 \\
\hline Group b & 20 & 50 \\
\hline Group c & 30 & 50 \\
\hline Group d & 50 & 50 \\
\hline Group e & 30 & 30 \\
\hline Group $\mathrm{f}$ & 50 & 30 \\
\hline Group g & 100 & 30 \\
\hline
\end{tabular}

Figure 1. Local manipulation of EP on the knee joint and group details. (a) Electrode pads were placed on the medial and lateral joint space of OA rats. (b) Detailed parameters of each group.

GGC-3' and reverse primer 5'-CTGGCTCAGCCACTCCAGC-3'; rat ADAMTS-4 forward primer 5'-AGA GTCCGAACGAGTTTACG-3' and reverse primer 5'-GTGCCAGTTCTGTGCGTC-3'; rat MMP-13 forward primer 5'-CCCAGCCCTATCCCTTGATGCCA-3' and reverse primer 5'-TGCAGGCGCCAGAAGAATCTG T-3'. PCR amplification reactions were run in a total volume of $20 \mu \mathrm{L}$. The reaction conditions were as follows: enzyme activation at $95^{\circ} \mathrm{C}$ for $1 \mathrm{~min}$, followed by 40 cycles of denaturation at $95^{\circ} \mathrm{C}$ for $15 \mathrm{~s}$, and all the primers annealing temperatures are $57^{\circ} \mathrm{C}$ for $15 \mathrm{~s}$ and extension at $72^{\circ} \mathrm{C}$ for $45 \mathrm{~s}$. Results were recorded and analyzed using StepOne software v2.1 (Applied Biosystems, USA). Expression was normalized relative to GAPDH. Each experimental group consisted of three knee joints, and was repeated three times for each time point.

\subsection{Micro-CT Reconstruction}

A resolution microtomograph scanner was used to assess both qualitative and quantitative changes to the subchondral bone at each time point following induction of OA. First, rat knee joints fixed in $4 \%$ neutral paraformaldehyde were loaded into a Micro-CT holder. X-ray parameters were then manually adjusted to values at which mineralized tissue could be visualized. The joints were scanned a $360^{\circ}$ rotation at a $45 \mu \mathrm{m}$ resolution. A MicroView 3D image viewer (GE Healthcare, USA) was used to reconstruct the scan data and visualize the trabecular and subchondral bone. A cylindrical region of interest (ROI) was selected from the weight-bearing area of the medial femoral condyles for analysis. The volume and diameter of the trabecular bone were measured as trabecular thickness (Tb.Th), mean trabecular separation (Tb.Sp), bone volume per tissue volume (BV/TV), and bone mineral density (BMD).

\subsection{Catwalk Gait Analysis}

The Catwalk equipment consisted of an enclosed walkway with a glass platform that was illuminated by a completely internal reflection of green light. Whenever the rat's paw made contacted with the glass platform, green light was able to escape, and the area of illumination could be captured using Illuminated Footprint technology consisting of a high speed video camera positioned underneath the walkway. The home cage was located at the end of the walkway. Rats were placed in the entrance of the channel and allowed to walk freely across the glass into the home cage. Results were recorded and analyzed using the Catwalk XT 8.1 Software. The goal of this experimental approach was to measure 3D footprint intensity, which consists of all four paws within an individual frame. Intensities ranged from 0 to 225 and were identified by different colors. Differences in intensity are considered representative of movement-evoked pain in OA.

\subsection{Histological Examination and Immunohistochemistry of Rat Knee Joints}

Isolated knee joints were prepared for histological examination and immunohistochemistry (IHC). First, each joint sample was photographed for evaluation of cartilage repair. After gross examination, tissues were fixed in $4 \%$ neutral paraformaldehyde for 7 days, and decalcified in $10 \%$ neutral ethylene diamine tetraacetic acid (EDTA) for 4 weeks at $37^{\circ} \mathrm{C}$. Then, the samples were dehydrated using an alcohol gradient and embedded in paraffin blocks. Finally, samples were cut into $7 \mathrm{~mm}$ sections using a microtome (Leica Microsystems, Germa- 
ny). Representative sections were stained with hematoxylin and eosin (H-E) and Safranin orange for histological analysis.

Rat articular cartilage sections were incubated with rabbit monoclonal anti-hIL1Ra antibody (Abcam, ab124962) or rabbit anti-COL II antibody (Abcam, ab34712) overnight at $4^{\circ} \mathrm{C}$, and then incubated with goat anti-rabbit secondary antibodies (MaxVison, 150304407E). Stained samples were photographed digitally and observed under confocal microscopy (Olympus, Japan).

\subsection{Statistical Analyses}

All quantitative date are expressed as mean \pm SD. A Tukey test was used to assess differences between groups. $P$ values $\leq 0.05$ were considered statistically significant.

\section{Results}

\subsection{Optimization of EP Parameters for the Intra-Articular Delivery of Plasmids in Rats}

Few previous studies have described transgene expression after plasmid transfer to the joint by EP [32]-[34], in those studies, have not statement the details of variables about plus width and plasmid dose, therefore, we sought to optimize EP parameters for the intra-articular delivery of plasmids in rats. Parameter optimization focused on pulse width and plasmid dose, with various levels of each parameter divided into seven experimental groups (Figure 1(b)). Plasmid dose was performed at two different levels, and pulse width at five different levels spanning the entire range of the EP equipment. Fluorescence was acquired up to 28 days after electroporation. Intense fluorescence activity was observed in the right knee joint 1 day after EP. The strongest activity was seen in group C (50 $\mu \mathrm{g}, 30 \mathrm{~ms})$, with significantly higher counts per second (CPS) levels, as compared with other groups. Furthermore, this elevated activity level was maintained over the course of the study (up to 28 days). The intensity of luciferase activity increased in response to higher plasmid concentrations under stable pulse width conditions. The degree of fluorescence intensity also increased in response to increasing pulse width when plasmid concentrations were held constant, but began to decline as pulse width (over $30 \mathrm{~ms}$ ) were increased (Figure 2(a)). Based upon these results, a combination of $50 \mu \mathrm{g}$ plasmid pulsed for $30 \mathrm{~ms}$ ensured high trans-

(a)

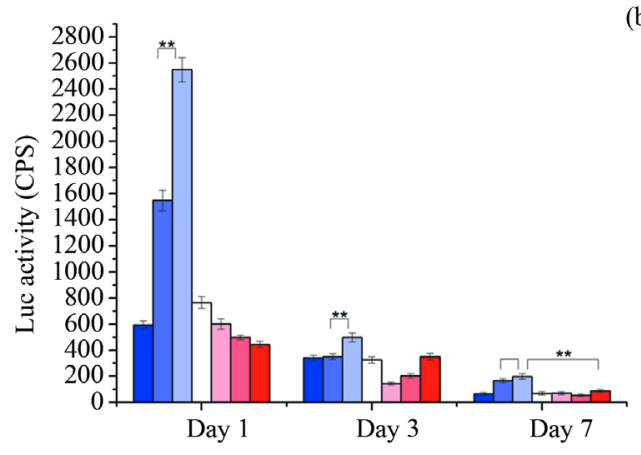

(b)

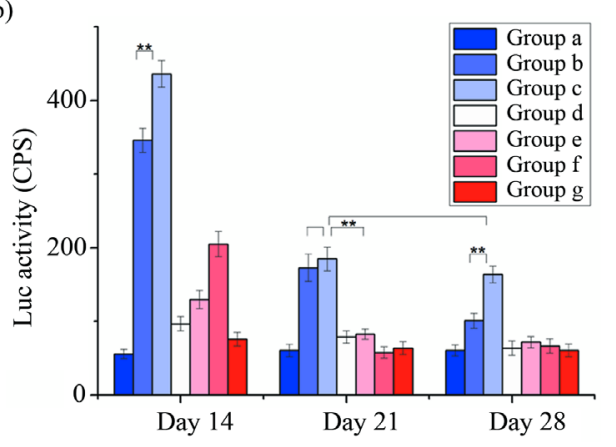

(c)

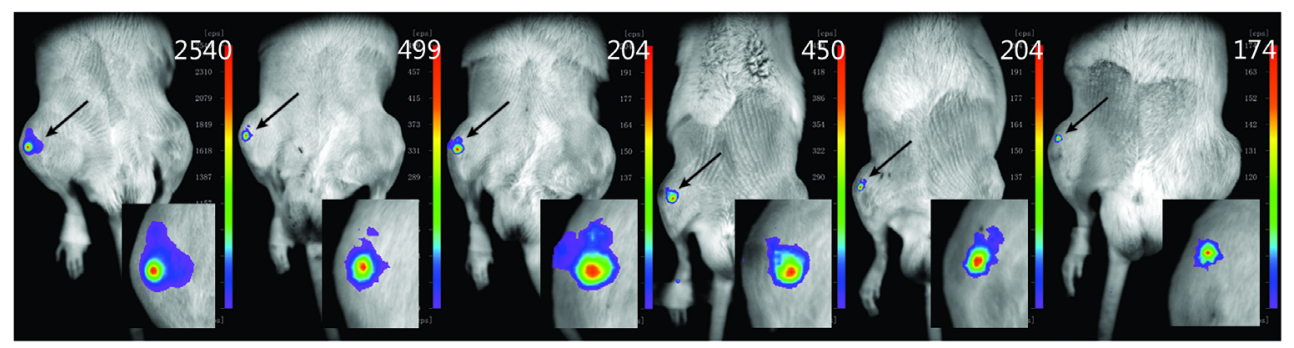

Figure 2. Bioluminescence imaging of transfected Luc in rat joints. (a), (b) BLI was used to monitor luciferase activity in knee joints following pLuc injection and EP. The $\mathrm{x}$ axis indicates the time post-electroporation; the $\mathrm{y}$ axis shows Luc activity in CPS. ${ }^{*} \mathrm{p}<0.05$ and ${ }^{* *} \mathrm{p}<0.01$, Tukey test, $\mathrm{n}=3$. (c) Respective pictures of group c at each time point. EP, electroporation; BLI, bioluminescence imaging; CPS, counts per second. 
fection efficiency of EP. Bioluminiesence signals were clearly observed in all groups, with representative images of group C (50 $\mu \mathrm{g}, 30 \mathrm{~ms})$ shown in Figure 2(c) for each time point.

\subsection{Development of Inflammatory-Induced Gait Adaptations: Catwalk Test}

The mean intensity (weight load) of the right hind paw while walking decreased significantly over the total test period in the both OA and NP groups (naked plasmid (NP) group) relative to the normal group (Table 1). In the NP group, light intensity was significantly higher $(\mathrm{p}=0.042)$ than the OA group one week post-electroporation; however, no significant differences between the OA and NP groups were observed at subsequent time points. No significant differences were observed in the light intensity of either the EP or AAV groups over time when compared with the normal group. Within the normal group, intensity increased as a function of time due to normal rat growth, resulting in the paw exerting more pressure on the walkway. Mean intensity was also significantly higher in the EP group relative to the NP group. The effect of the human IL-1Ra plasmid depended on whether EP occurred after plasmid injection, with significant improvements in the weight load of the right joint seen in the EP group across the entire test period (Table 1). At 4 weeks post-treatment, 3D Footprint Intensity pictures (Figure 3) showed a strong correlation with the results shown in Table 1. There were multiple 3D peak in the results of EP group (Figure 3(c)) and AAV group (Figure 3(d)), each representative of different parts of the rats plantar pressure on the treadmill, in these two groups respectively, the number of peaks are superfluous OA group (Figure 3(a)) and NP group (Figure 3(b)).

\subsection{Transgene Expression Post-Treatment in the Knee Joint}

To examine transfection efficiency and explore interconnectedness of OA associated inflammatory factors, we determined the expression level of hIL-1Ra, IL-1 $\beta$, TNF- $\alpha$, MMP-13, and ADAMTS-4 in extracts from rat knee joint using PCR analysis. mRNA expression of IL-1Ra was significantly up-regulated in both the EP and AAV groups relative to other groups at each time point (Figure 4(a)). Expression of IL-1Ra did decrease over time; however, these levels were still maintained at levels $\sim 4$-fold higher than those of controls at 4 weeks. Over the first two weeks, IL-1Ra levels in the AAV group were significantly higher than those of the EP group, with the opposite results demonstrated in the last two weeks, indicating that AAV shows higher transfection efficiency

(a)

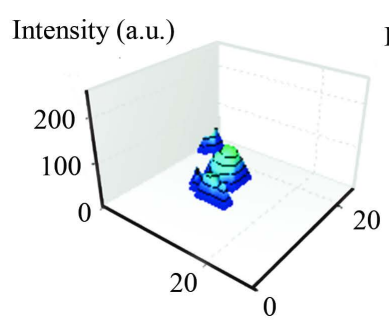

(b)

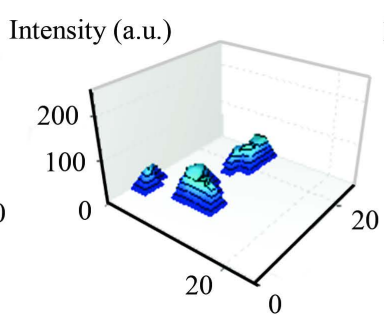

(c)

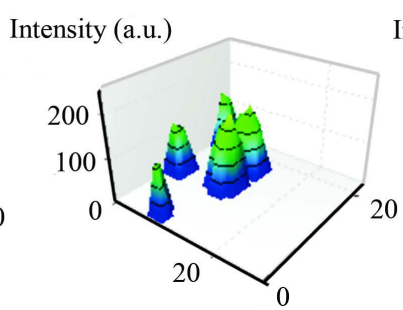

(d)

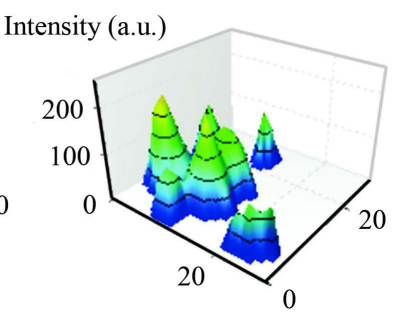

Figure 3. Catwalk analysis depicting 3D footprint intensities of a representative rat crossing the walkway at 4 weeks post-treatment; the y axis shows intensity in a.u. (a) OA group. (b) NP group. (c) EP group. (d) AAV group. a.u., arbitrary units.

Table 1. Effects on changes in weight load (intensity; a.u.) caused by arthritis.

\begin{tabular}{ccccc}
\hline Group & $1 \mathrm{~W}$ & $2 \mathrm{~W}$ & $3 \mathrm{~W}$ & $4 \mathrm{~W}$ \\
\hline OA & $103.94 \pm 1.45^{*}$ & $99.04 \pm 3.62^{*}$ & $78.24 \pm 1.29^{*}$ & $66.56 \pm 5.66^{*}$ \\
NP & $113.79 \pm 5.60^{* \#}$ & $93.15 \pm 3.34^{*}$ & $76.89 \pm 1.19^{*}$ & $68.72 \pm 2.07^{*}$ \\
EP & $124.30 \pm 2.26^{\# \Delta}$ & $120.80 \pm 2.01^{* \# \Delta}$ & $118.79 \pm 0.60^{* \# \Delta}$ & $119.25 \pm 3.28^{* \# \Delta}$ \\
AAV & $124.88 \pm 3.02^{\# \Delta}$ & $128.35 \pm 4.41^{\# \Delta}$ & $117.57 \pm 4.65^{* \# \Delta}$ & $110.50 \pm 2.71^{* \# \Delta}$ \\
Control & $127.58 \pm 1.61$ & $130.00 \pm 2.05$ & $136.70 \pm 6.97$ & $139.66 \pm 5.34$
\end{tabular}

Values are mean \pm SD. Repeated measures ANOVA, with Tukey test used compared with normal rats, ${ }^{*}$ p $<0.05$; compared with OA group rats, ${ }^{*}$ p $<$ 0.05 ; compared with NP group rats, ${ }^{\Delta} \mathrm{p}<0.05$, at each time point. 
(a)

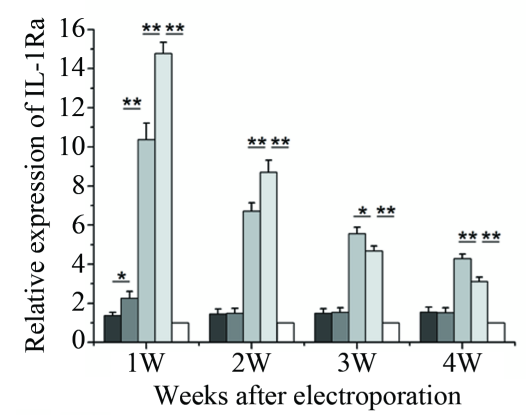

(d)

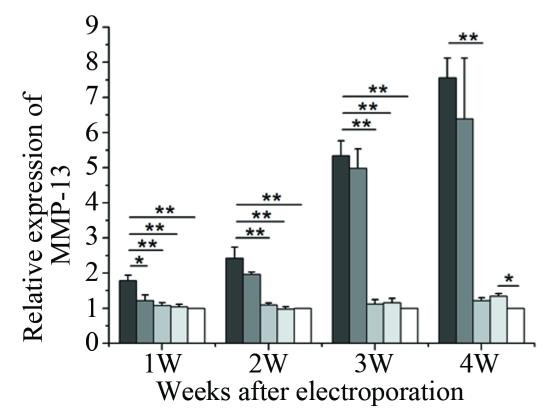

(b)

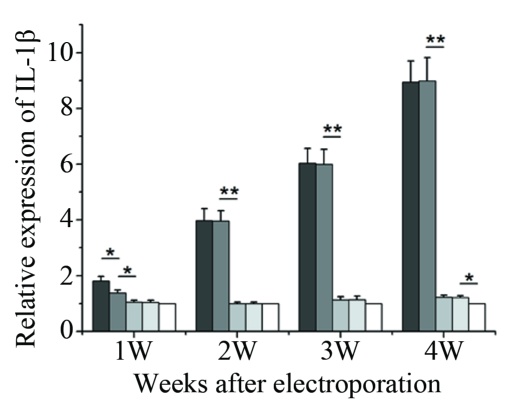

(e)

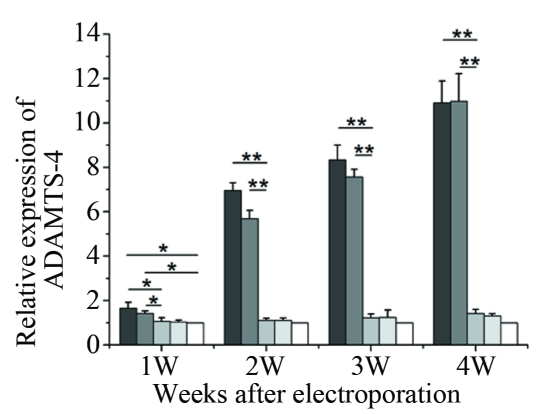

(c)

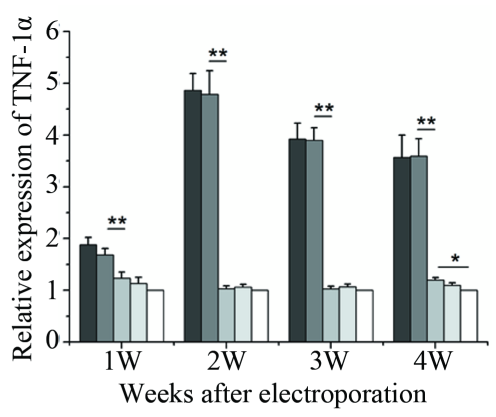

Figure 4. Relative gene expression of (a) IL-1Ra, (b) IL-1 $\beta$, (c) TNF- $\alpha$, (d) MMP-13, (e) ADAMTS-4 in OA articular cartilage and synovium analyzed by RT-PCR. ${ }^{*} \mathrm{p}<0.05$ and ${ }^{* *} \mathrm{p}<0.01$.

than EP, but is more susceptible to degradation and inactivation. mRNA expression was significantly higher in the NP group relative to both the OA and normal groups at 1 week; no statistically significant differences were observed at any subsequent time points, suggesting that the naked plasmid was not the source of any desired therapeutic effect.

Consistent with its role as the primary factor in the OA inflammatory cascade, IL-1 $\beta$ expression gradually increased in OA rats throughout the course of this experiment. In both the EP and AAV groups, IL- $1 \beta$ mRNA expression was higher than that of the normal group, though significant differences did not appear until week 4 . In the NP group, IL-1 $\beta$ mRNA levels were significantly different compared to the OA group at 1 week. Naked IL-1Ra plasmid was able to confer modest suppression of inflammation at week 1, but the efficiency of this treatment was quickly lost (Figure 4(b)).

TNF- $\alpha$ mRNA levels were strongly correlated with an increase in OA-mediated joint swelling [35]. Peak TNF- $\alpha$ expression was observed in both the OA and NP groups at 2 weeks and decreased thereafter, though mRNA levels were still significantly higher than those of normal controls (Figure 4(c)). Due to the effects of EP and AAV transduction, TNF- $\alpha$ expression levels remained at levels similar to those of the control group, though significant differences between the EP and normal groups were detected by week 4 .

MMP-13 and ADAMTS-4 are important downstream targets of both IL- $1 \beta$ and TNF- $\alpha$ [36]. MMP-13 and ADAMTS-4 mRNA expression increased over time in both the OA and NP groups, while no significant differences in expression levels were seen in either treatment group. The only exception was the EP group at week 4, which showed higher levels of MMP-13 relative to the normal group $(\mathrm{P}<0.05)$. Overall, variations in MMP-13 and ADAMTS-4 expression were similar to this of IL-1 $\beta$ (Figure 4(d) and Figure 4(e)).

\subsection{Structural Analysis of the Subchondral Bone at the Medial Femoral Condyle}

To better understand the pathology of trauma-induced OA in rats, we used Micro-CT to analyze changes in the subchondral bone. In sites of severe arthritis, the subchondral bone becomes thicker [37]. Among the untreated OA groups, BMD, BV/TV, and Tb.Th all show gradual increases over time, with these three parameters increasing 4, 3, and 6-fold, respectively, relative to the normal group at 4 weeks (Figures 5(a)-(c)). With respect 

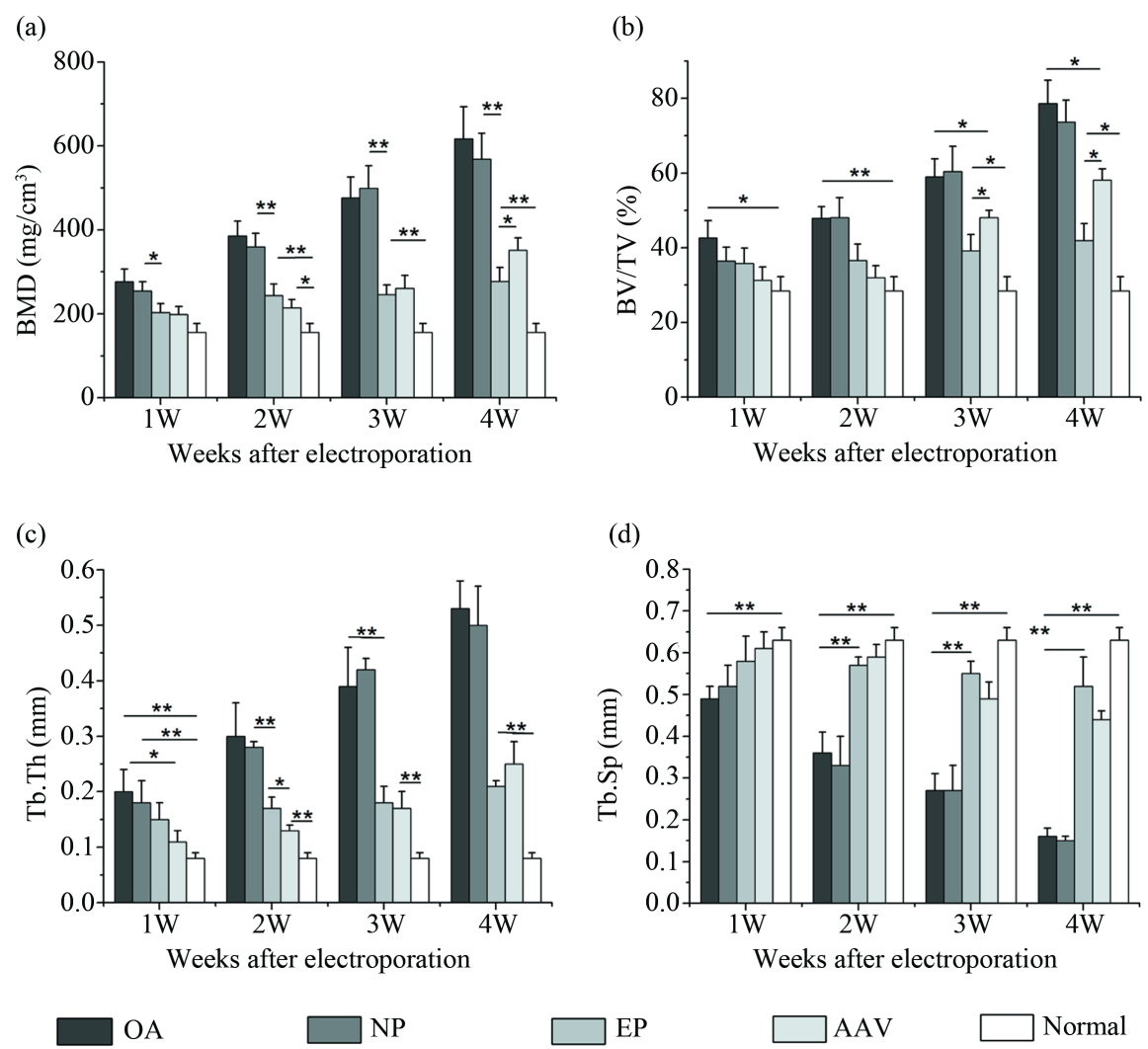

Figure 5. Changes in bone histomorphometry caused by arthritis. (a) BMD, (b) BV/TV, (c) Tb.Th, (d) Tb.Sp. ${ }^{*} \mathrm{p}<0.05$ and ${ }^{* *} \mathrm{p}<0.01$.

to each of these parameters, no significant differences were seen between the OA and NP groups, though all were significantly higher than the normal group, the only exception being the BV/TV score in the NP group, which was not significantly different from the normal group at 1 week. In contrast, each of these values were significantly lower in both the EP and AAV groups, relative to the OA and NP groups over the entire test period. While all three of these values did increase over time, the rate of increase was significantly lower than that of either the OA or NP groups (Figure 5). These results showed that both EP and AAV transduction were able to suppress the majority of subchondral bone remodeling and may, therefore, delay the development of osteoarthritis [38].

\subsection{Gross Morphology and Histological Evaluation}

Gross evidence of cartilage damage was assessed in each of the five groups at 4 weeks after treatments (Figure 6). Medial femoral condyle cartilage in the OA and NP groups exhibited serious defects, including an irregular surface, with accumulation seen across virtually the entire weight-bearing area; subchondral bone was exposed in the seriously damaged regions (bleed exudation). Significantly better results were observed in the AAV and EP groups, with the medial femoral condyle surface covered by flat, glossy, and smooth cartilage, with no evidence of macroscopic cartilage degeneration. In the Figure 6, the "Normal" is the results of control group which shows the histological evaluation of cartilage.

To evaluate the cartilage damage, histological staining was done 1, 2, 3 and 4 weeks after treatment (Figure 6). In the OA and NP groups, the superficial surface layer of articular cartilage had become coarse and layered, with the extracellular matrix slightly degraded 1 week after treatment (Figure 6A, Figure 6B, Figure 6a, Figure 6b). Gradual increases in damage to the articular cartilage, decreases in proteoglycan levels, and chondrocyte disarrangement were evident, along with an influx of cell clusters in the intermediate layer (Figure 6I and Figure 6J). Proteoglycan was not uniformly distributed, and characterized by de-stained regions, particularly in the areas surrounding cell clusters (Figure 6F, Figure 6I, Figure 6j, Figure 6m, Figure 6n). By week 4 post- 


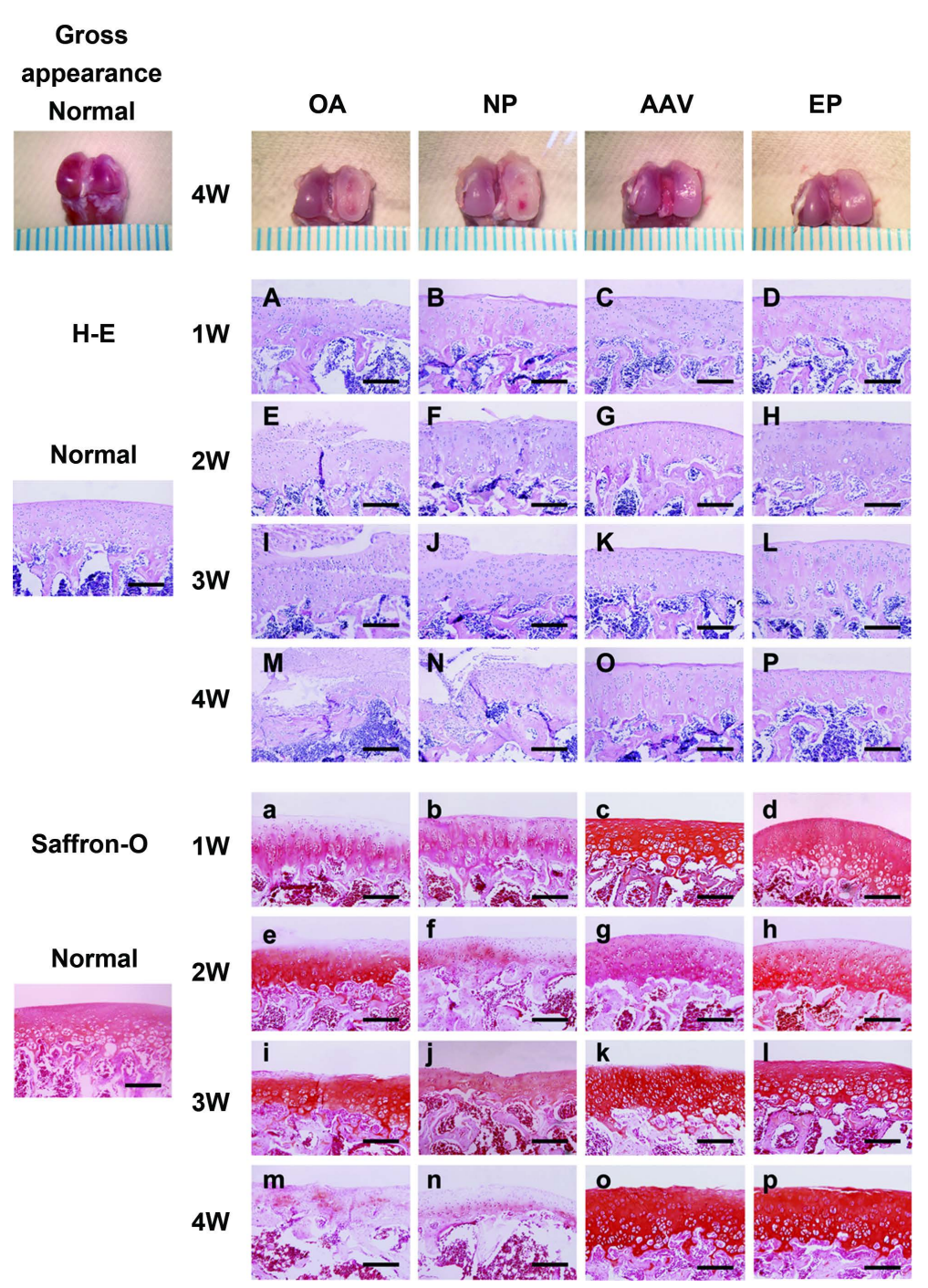

Figure 6. Histological evaluation of cartilage in four groups 4 weeks after treatment. Paraffin sections were stained with hematoxylin and eosin (A-P) and Safranin O (a-p). (A, E, I, M, a, e, i, m) Untreated, OA group; (B, F, J, N, b, f, j, n) Osteoarthritis with hIL-1Ra plasmid treatment, NP group; (C, G, K, O, c, g, k, o) Osteoarthritis with AAV-hIL-1Ra treatment, AAV group; (D, H, L, $\mathrm{P}, \mathrm{d}, \mathrm{h}, \mathrm{l}, \mathrm{p})$ Osteoarthritis and plasmid with electroporation, EP group. 200 times magnification.

treatment, crack depth in the subchondral bone and chondrocytes had become extremely disordered, with the cartilage almost completely destroyed, and the subchondral bone plate shifted (Figure 6M, Figure 6N, Figure $6 \mathrm{~m}$, Figure $6 \mathrm{n}$ ). These pathological changes were much less severe in both the AAV and EP groups relative to the OA and NP groups. The surface of the cartilage in the AAV and EP groups remained smooth for at least 3 weeks after treatment (Figure 6K, Figure 6L, Figure 6k, Figure 6l). By week 4, the superficial surface layer of articular cartilage had been lost, but this degradation did not extend into the extracellular matrix (Figure 60, Figure 6P, Figure 60 and Figure 6p).

In the Figure 7, the "Normal" is the results of control group which presents immunohistochemical staining of cartilage. IL-1Ra was transfected by EP and AAV into the articular cartilage. The integrated cartilage was strongly positive for IL-1Ra in both the AAV and EP groups, with significant differences seen relative to the OA and NP groups at each time point (Figures 7A-P). These high levels of IL-1Ra expression were maintained for at least 4 weeks after treatment (Figure 70 and Figure 7P). Despite serious damage to the cartilage in both the OA and NP groups at 4 weeks, the remaining cartilage was positive for IL-1Ra at levels higher than that of the first three weeks (Figure 7A, Figure 7B, Figure 7E, Figure 7F, Figure 7I, Figure 7J, Figure 7M, Figure 7N), 


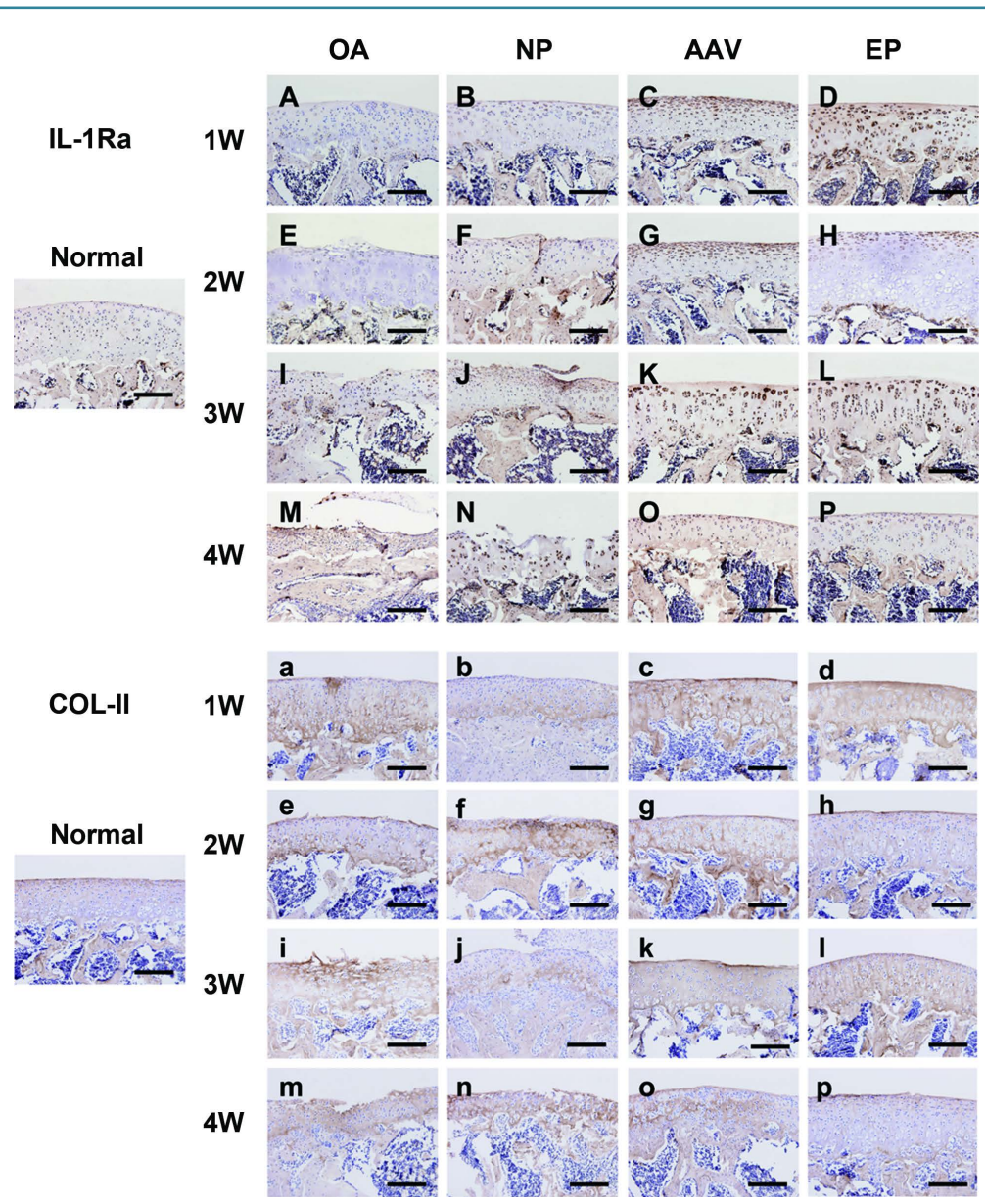

Figure 7. Immunohistochemical staining of cartilage following treatment with IL-1Ra (A-P) and type II collagen (a-p). (A, E, I, M, a, e, i, m) Untreated, OA group; (B, F, J, N, b, f, j, n) osteoarthritis with hIL-1Ra plasmid treatment, NP group; (C, G, K, O, c, g, k, o) osteoarthritis with AAV-hIL-1Ra treatment, AAV group; (D, H, L, P, d, h, l, p) osteoarthritis and plasmid with electroporation, EP group. 200 times magnification.

indicating a possible up-regulation of IL-1Ra at this time point. As the major collagen present in the chondrocyte extracellular matrix, type II collagen was gradually degraded during OA progression (Figure 7a, Figure 7e, Figure 7i, Figure 7m) [21] [22]. In the AAV and EP groups, intact cartilage was hard to differentiate from normal cartilage based upon type II collagen immunohistochemical staining (Figure 7c, Figure 7d, Figure 7g, Figure 7h, Figure 7k, Figure 7l), whereas the type II collagen was weakly positive at 4 weeks post-treatment compared with normal cartilage (Figure 70 and Figure 7p).

\section{Discussion}

OA progression is a complex process that includes multiple cellular and molecular events that take place in a specific spatial and temporal order. Regulation of inflammatory processes in arthritis is driven in part by IL-1 and its natural inhibitor IL-1Ra [19] [20] [24]. The effectiveness of IL-1Ra as a treatment for OA has been demonstrated both ex vivo and in vivo, resulting in a significant inhibition of IL-1-induced rabbit arthritis [39] [40], as well as protecting horse forelimb joint cartilage from OA [41].

The data presented here show that IL-1Ra can be successfully transduced in vivo, overcoming important technical challenges in terms of safety, efficacy, and convenience for transgene delivery [8] [15] [41]. EP delivery of IL-1Ra offers a virus-free approach to transgene delivery, which has been validated in the context of bone formation and for the inhibition rheumatoid arthritis [29] [31]. However, no attempts have been made to utilize in vivo $\mathrm{EP}$ for OA treatment. 
Initial validation of EP for intra-articular delivery required optimization of delivery parameters for both pulse width and plasmid dose. In vivo bioluminescence imaging was used to detect the fluorescence following transfection with a plasmid encoding luciferase [42]. Using this method, our data showed a combination of $50 \mu \mathrm{g}$ plasmid pulsed for $30 \mathrm{~ms}$ as the optimal combination for maximizing transfection efficiency (Figure 2).

AAV-mediated delivery is a well-established model for gene therapy, having been studied in numerous diseases [8] [43] [44], making it an ideal control for evaluating the efficacy of EP. Initial in vivo viral gene therapy studies revealed poor transduction into the cartilage [8] [45] [46], probably as a result of the viral diameter, which is often too big to penetrate the cartilage matrix [47]. However, a study by Goodrich et al. were able to achieve strong transduction of equine joints injected with scAAV-GFP, with GFP fluorescence detected within the cartilage at least 98 days after injection [43]. The data presented here are broadly consistent with the latter result, with high IL-1Ra levels detected in the cartilage 4 weeks after treatment for both the AAV and EP groups (Figure 70 and Figure 7P), indicating efficient transduction of the joint cartilage.

A careful analysis of effects of OA inhibition is indispensable for assessing the success of EP as a transfection technique. In this study, both $\mu \mathrm{CT}$ and histological analyses revealed progressive destruction of the medial femoral condyle cartilage and subchondral bone induced by OA. Catwalk gait analysis was used to correlate joint damage with movement-evoked pain in OA.

$\mathrm{BMD}, \mathrm{BV} / \mathrm{TV}$, Tb.Th scores obtained for the subchondral bone underlying the weight-bearing areas of the medial femoral condyle were significantly lower in the EP and AAV groups relative to untreated OA controls. Taken together, these data demonstrate similar efficacy for both EP and AAV-mediated delivery of IL-1Ra in terms of delay the remodeling of subchondral bone. While the timing and relationship between cartilage destruction and subchondral bone reconstruction remains poorly understood [38] [48], evidence of a gene therapy-based treatment for OA represents a promising development for patients suffering from OA [37].

Little difference is seen in terms of $\mu \mathrm{CT}$ analysis of the subchondral bone or expression of inflammatory markers of transfected mice, including IL-1 $\beta$, TNF- $\alpha$, MMP-13, and ADAMTS-4 over the first three weeks. While none of these factors were expressed at levels significantly higher than that of normal controls, both BMD and $\mathrm{Tb}$.Th were, implying that the subchondral bone reconstruction may be occurring prior to inflammation of the cartilage and synovium.

After electroporation of IL-1Ra into the articular, inflammatory related factors IL- $1 \beta$, TNF- $\alpha$, MMP-13, and ADAMTS- 4 were all strongly downregulated. IL- $1 \beta$ has been shown to induce chondrocytes to produce metalloproteinases, mainly interstitial collagenase 3 (MMP-13), a family of enzymes that play an important role in the degradation of cartilage components [49] [50]. Catabolic cytokines such as IL- $1 \beta$ and TNF- $\alpha$ induce chondrocytes to produce ADAMTS-4, which is responsible for the proteolysis of aggrecan molecules [36]. Members of both the MMP and ADAMTS families play a central role in aggrecan degradation in arthritis [36] [49] [50]. Taken together, transfection of IL-1Ra into the OA articular not only inhibits the function of IL-1 itself but also limits the potential downstream effects of IL- 1 and TNF- $\alpha$, providing a multi-faceted therapeutic effect in OA.

Continued expression of target gene IL-1Ra was seen in both the AAV and EP groups; however, a sharp decrease was seen in the AAV group compared with that of the EP at four weeks post-treatment [43], suggesting that EP may provide more persistent gene expression. Further studies will examine the duration of hIL-1Ra expression following transfection by EP using an observation period significantly longer than used in our initial trial. A recent publication supports the notion that in vivo electroporation may be applicable in large animal models and, subsequently, human trials [51]; a similar test of clinically efficacy on hIL-1Ra in a large animal model should therefore be performed.

\section{Conclusion}

The use of EP as an efficient method for therapeutic gene delivery into the intro-articular tissue, with gene expression levels similar to that of AAV, with expression for longer periods of time. Given the well-documented safety issues associated with AAV delivery, EP may constitute an effective alternative for gene delivery in OA.

\section{Acknowledgements}

Funding: This work was funded by Ministry of science and technology project 973(2014CB542201); Beijing municipal science and technology projects (Z141107004414044); National high technology research and development program of 863 (2012AA020502); National Natural Science Foundation of China (81572148). 
Financial interest: There is no conflict of interest in this section.

The authors gratefully acknowledge the assistance of Feng $\mathrm{Xu}$ of the department of Orthopedics, General Hospital of People's Liberation Army, Beijing, People’s Republic of China, for their contribution in surgery procedures.

\section{References}

[1] Iannone, F. and Lapadula, G (2003) The Pathophysiology of Osteoarthritis. Aging Clinical and Experimental Research, 15, 364-372. http://dx.doi.org/10.1007/BF03327357

[2] Mankin, H.J., Johnson, M.E. and Lippiello, L. (1981) Biochemical and Metabolic Abnormalities in Articular Cartilage from Osteoarthritic Human Hips. III. Distribution and Metabolism of Amino Sugar-Containing Macromolecules. The Journal of Bone and Joint Surgery American Volume, 63, 131-139.

[3] Zhang, X.L., Yu, C.L., Shi, X., Zhang, C., Tang, T.T. and Dai, K.R. (2006) Direct Chitosan-Mediated Gene Delivery to the Rabbit Knee Joints in Vitro and in Vivo. Biochemical and Biophysical Research Communications, 341, 202-208. http://dx.doi.org/10.1016/j.bbrc.2005.12.171

[4] Evans, C.H., Robbins, P.D., Ghivizzani, S.C., Wasko, M.C., Tomaino, M.M., Kang, R., et al. (2005) Gene Transfer to Human Joints: Progress toward a Gene Therapy of Arthritis. Proceedings of the National Academy of Sciences of the United States of America, 102, 8698-8703. http://dx.doi.org/10.1073/pnas.0502854102

[5] Lang, A., Neuhaus, J., Pfeiffenberger, M., Schroder, E., Ponomarev, I., Weber, Y., et al. (2014) Optimization of A Nonviral Transfection System to Evaluate Cox-2 Controlled Interleukin-4 Expression for Osteoarthritis Gene Therapy in Vitro. The Journal of Gene Medicine, 16, 352-363. http://dx.doi.org/10.1002/jgm.2812

[6] Madry, H. and Cucchiarini, M. (2014) Tissue-Engineering Strategies to Repair Joint Tissue in Osteoarthritis: Nonviral Gene-Transfer Approaches. Current Rheumatology Reports, 16, 450. http://dx.doi.org/10.1007/s11926-014-0450-7

[7] Lu, H.D., Zhao, H.Q., Wang, K. and Lv, L.L. (2011) Novel Hyaluronic Acid-Chitosan Nanoparticles as Non-Viral Gene Delivery Vectors Targeting Osteoarthritis. International Journal of Pharmaceutics, 420, 358-365. http://dx.doi.org/10.1016/j.ijpharm.2011.08.046

[8] Goodrich, L.R., Phillips, J.N., McIlwraith, C.W., Foti, S.B., Grieger, J.C., Gray, S.J., et al. (2013) Optimization of scAAVIL-1ra in Vitro and in Vivo to Deliver High Levels of Therapeutic Protein for Treatment of Osteoarthritis. Molecular Therapy Nucleic Acids, 2, e70. http://dx.doi.org/10.1038/mtna.2012.61

[9] Chiu, W.C., Chen, C.M., Cheng, T.T., You, H.L., Yu, S.F., Weng, L.H., et al. (2013) EBV-Encoded Small RNA1 and Nonresolving Inflammation in Rheumatoid Arthritis. The Kaohsiung Journal of Medical Sciences, 29, 606-610. http://dx.doi.org/10.1016/j.kjms.2013.04.002

[10] Croia, C., Serafini, B., Bombardieri, M., Kelly, S., Humby, F., Severa, M., et al. (2013) Epstein-Barr Virus Persistence and Infection of Autoreactive Plasma Cells in Synovial Lymphoid Structures in Rheumatoid Arthritis. Annals of the Rheumatic Diseases, 72, 1559-1568. http://dx.doi.org/10.1136/annrheumdis-2012-202352

[11] Hsieh, J.L., Shen, P.C., Shiau, A.L., Jou, I.M., Lee, C.H., Teo, M.L., et al. (2009) Adenovirus-Mediated Kallistatin Gene Transfer Ameliorates Disease Progression in a Rat Model of Osteoarthritis Induced by Anterior Cruciate Ligament Transection. Human Gene Therapy, 20, 147-158. http://dx.doi.org/10.1089/hum.2008.096

[12] Simon, R.H., Engelhardt, J.F., Yang, Y., Zepeda, M., Weber-Pendleton, S., Grossman, M., et al. (1993) AdenovirusMediated Transfer of the CFTR Gene to Lung of Nonhuman Primates: Toxicity Study. Human Gene Therapy, 4, 771780. http://dx.doi.org/10.1089/hum.1993.4.6-771

[13] Rothe, M., Modlich, U. and Schambach, A. (2013) Biosafety Challenges for Use of Lentiviral Vectors in Gene Therapy. Current Gene Therapy, 13, 453-468. http://dx.doi.org/10.2174/15665232113136660006

[14] Heckl, D., Schwarzer, A., Haemmerle, R., Steinemann, D., Rudolph, C., Skawran, B., et al. (2012) Lentiviral Vector Induced Insertional Haploinsufficiency of Ebf1 Causes Murine Leukemia. Molecular Therapy: The Journal of the American Society of Gene Therapy, 20, 1187-1195.

[15] Gao, Y., Xu, Z., Chen, S., Gu, W., Chen, L. and Li, Y. (2008) Arginine-Chitosan/DNA self-Assemble Nanoparticles for Gene Delivery: In Vitro Characteristics and Transfection Efficiency. International Journal of Pharmaceutics, 359, 241-246. http://dx.doi.org/10.1016/j.ijpharm.2008.03.037

[16] Hejazi, R. and Amiji, M. (2003) Chitosan-Based Gastrointestinal Delivery Systems. Journal of Controlled Release, 89, 151-165. http://dx.doi.org/10.1016/S0168-3659(03)00126-3

[17] Li, Z. and Zhang, M. (2005) Chitosan-Alginate as Scaffolding Material for Cartilage Tissue Engineering. Journal of Biomedical Materials Research Part A, 75, 485-493. http://dx.doi.org/10.1002/jbm.a.30449

[18] Mao, S., Sun, W. and Kissel, T. (2010) Chitosan-Based Formulations for Delivery of DNA and siRNA. Advanced Drug Delivery Reviews, 62, 12-27. http://dx.doi.org/10.1016/j.addr.2009.08.004 
[19] Pelletier, J.P., Faure, M.P., DiBattista, J.A., Wilhelm, S., Visco, D. and Martel-Pelletier, J. (1993) Coordinate Synthesis of Stromelysin, Interleukin-1, and Oncogene Proteins in Experimental Osteoarthritis. An Immunohistochemical Study. The American Journal of Pathology, 142, 95-105.

[20] Goldring, M.B. (1999) The Role of Cytokines as Inflammatory Mediators in Osteoarthritis: Lessons from Animal Models. Connective Tissue Research, 40, 1-11. http://dx.doi.org/10.3109/03008209909005273

[21] Zhu, S., Lu, P., Liu, H., Chen, P., Wu, Y., Wang, Y., et al. (2015) Inhibition of Rac1 Activity by Controlled Release of NSC23766 from Chitosan Microspheres Effectively Ameliorates Osteoarthritis Development in Vivo. Annals of the Rheumatic Diseases, 74, 285-293. http://dx.doi.org/10.1136/annrheumdis-2013-203901

[22] Bondeson, J., Wainwright, S.D., Lauder, S., Amos, N. and Hughes, C.E. (2006) The Role of Synovial Macrophages and Macrophage-Produced Cytokines in Driving Aggrecanases, Matrix Metalloproteinases, and Other Destructive and Inflammatory Responses in Osteoarthritis. Arthritis Research \& Therapy, 8, R187. http://dx.doi.org/10.1186/ar2099

[23] Goldring, M.B., Sandell, L.J., Stephenson, M.L. and Krane, S.M. (1986) Immune Interferon Suppresses Levels of Procollagen mRNA and Type II Collagen Synthesis in Cultured Human Articular and Costal Chondrocytes. The Journal of Biological Chemistry, 261, 9049-9055.

[24] Eisenberg, S.P., Evans, R.J., Arend, W.P., Verderber, E., Brewer, M.T., Hannum, C.H., et al. (1990) Primary Structure and Functional Expression from Complementary DNA of a Human Interleukin-1 Receptor Antagonist. Nature, 343, 341-346. http://dx.doi.org/10.1038/343341a0

[25] Dhaneshwar, S., Dipmala, P., Abhay, H. and Prashant, B. (2013) Disease-Modifying Effect of Anthraquinone Prodrug with Boswellic Acid on Collagenase-Induced Osteoarthritis in Wistar Rats. Inflammation \& Allergy Drug Targets, 12, 288-295. http://dx.doi.org/10.2174/18715281113129990002

[26] Frisbie, D.D., Ghivizzani, S.C., Robbins, P.D., Evans, C.H. and McIlwraith, C.W. (2002) Treatment of Experimental Equine Osteoarthritis by in Vivo Delivery of the Equine Interleukin-1 Receptor Antagonist Gene. Gene Therapy, 9, 1220. http://dx.doi.org/10.1038/sj.gt.3301608

[27] Pelletier, J.P., Caron, J.P., Evans, C., Robbins, P.D., Georgescu, H.I., Jovanovic, D., et al. (1997) In Vivo Suppression of Early Experimental Osteoarthritis by Interleukin-1 Receptor Antagonist Using Gene Therapy. Arthritis and Rheumatism, 40, 1012-1019. http://dx.doi.org/10.1002/art.1780400604

[28] Choi, S.A., Hwang, S.K., Wang, K.C., Cho, B.K., Phi, J.H., Lee, J.Y., et al. (2011) Therapeutic Efficacy and Safety of TRAIL-Producing Human Adipose Tissue-Derived Mesenchymal Stem Cells against Experimental Brainstem Glioma. Neuro-Oncology, 13, 61-69. http://dx.doi.org/10.1093/neuonc/noq147

[29] Kimelman-Bleich, N., Pelled, G., Zilberman, Y., Kallai, I., Mizrahi, O., Tawackoli, W., et al. (2011) Targeted Geneand-Host Progenitor Cell Therapy for Nonunion Bone Fracture Repair. Molecular Therapy, 19, 53-59. http://dx.doi.org/10.1038/mt.2010.190

[30] Gehl, J. (2003) Electroporation, Theory and Methods, Perspectives for Drug Delivery, Gene Therapy and Research. Acta Physiologica Scandinavica, 177, 437-447. http://dx.doi.org/10.1046/j.1365-201X.2003.01093.X

[31] Sukedai, M., Ariyoshi, W., Okinaga, T., Iwanaga, K., Habu, M., Yoshioka, I., et al. (2011) Inhibition of Adjuvant Arthritis in Rats by Electroporation with Interleukin-1 Receptor Antagonist. Journal of Interferon \& Cytokine Research, 31, 839-846. http://dx.doi.org/10.1089/jir.2011.0024

[32] Grossin, L., Cournil-Henrionnet, C., Mir, L.M., Liagre, B., Dumas, D., Etienne, S., et al. (2003) Direct Gene Transfer into Rat Articular Cartilage by in Vivo Electroporation. FASEB Journal, 17, 829-835. http://dx.doi.org/10.1096/fj.02-0518com

[33] Bloquel, C., Trollet, C., Pradines, E., Seguin, J., Scherman, D. and Bureau, M.F. (2006) Optical Imaging of Luminescence for in Vivo Quantification of Gene Electrotransfer in Mouse Muscle and Knee. BMC Biotechnology, 6, 16. http://dx.doi.org/10.1186/1472-6750-6-16

[34] Ohashi, S., Kubo, T., Kishida, T., Ikeda, T., Takahashi, K., Arai, Y., et al. (2002) Successful Genetic Transduction in Vivo into Synovium by Means of Electroporation. Biochemical and Biophysical Research Communications, 293, 15301535. http://dx.doi.org/10.1016/S0006-291X(02)00386-8

[35] Seguin, C.A. and Bernier, S.M. (2003) TNFalpha Suppresses Link Protein and Type II Collagen Expression in Chondrocytes, Role of MEK1/2 and NF-KappaB Signaling Pathways. Journal of Cellular Physiology, 197, 356-369. http://dx.doi.org/10.1002/jcp.10371

[36] Verma, P. and Dalal, K. (2011) ADAMTS-4 and ADAMTS-5, Key Enzymes in Osteoarthritis. Journal of Cellular Biochemistry, 112, 3507-3514. http://dx.doi.org/10.1002/jcb.23298

[37] Madry, H., van Dijk, C.N. and Mueller-Gerbl, M. (2010) The Basic Science of the Subchondral Bone. Knee Surgery, Sports Traumatology, Arthroscopy, 18, 419-433. http://dx.doi.org/10.1007/s00167-010-1054-z

[38] Muraoka, T., Hagino, H., Okano, T., Enokida, M. and Teshima, R. (2007) Role of Subchondral Bone in Osteoarthritis Development: A Comparative Study of Two Strains of Guinea Pigs with and without Spontaneously Occurring Os- 
teoarthritis. Arthritis and Rheumatism, 56, 3366-3374. http://dx.doi.org/10.1002/art.22921

[39] Zhang, P., Zhong, Z.H., Yu, H.T. and Liu, B. (2015) Exogenous Expression of IL-1Ra and TGF-Beta1 Promotes in Vivo Repair in Experimental Rabbit Osteoarthritis. Scandinavian Journal of Rheumatology, 44, 404-411. http://dx.doi.org/10.3109/03009742.2015.1009942

[40] Chen, B., Qin, J., Wang, H., Magdalou, J. and Chen, L. (2010) Effects of Adenovirus-Mediated bFGF, IL-1Ra and IGF-1 Gene Transfer on Human Osteoarthritic Chondrocytes and Osteoarthritis in Rabbits. Experimental \& Molecular medicine, 42, 684-695. http://dx.doi.org/10.3858/emm.2010.42.10.067

[41] Watson, R.S., Broome, T.A., Levings, P.P., Rice, B.L., Kay, J.D., Smith, A.D., et al. (2013) ScAAV-Mediated Gene Transfer of Interleukin-1-Receptor Antagonist to Synovium and Articular Cartilage in Large Mammalian Joints. Gene Therapy, 20, 670-677. http://dx.doi.org/10.1038/gt.2012.81

[42] Buckley, S.M., Delhove, J.M., Perocheau, D.P., Karda, R., Rahim, A.A., Howe, S.J., et al. (2015) In Vivo Bioimaging with Tissue-Specific Transcription Factor Activated Luciferase Reporters. Scientific Reports, 5, Article Number: 11842. http://dx.doi.org/10.1038/srep11842

[43] Goodrich, L.R., Grieger, J.C., Phillips, J.N., Khan, N., Gray, S.J., McIlwraith, C.W., et al. (2015) ScAAVIL-1ra Dosing Trial in a Large Animal Model and Validation of Long-Term Expression with Repeat Administration for Osteoarthritis Therapy. Gene Therapy, 22, 536-545. http://dx.doi.org/10.1038/gt.2015.21

[44] Deng, W.T., Dyka, F.M., Dinculescu, A., Li, J., Zhu, P., Chiodo, V.A., et al. (2015) Stability and Safety of an AAV Vector for Treating RPGR-ORF15 X-Linked Retinitis Pigmentosa. Human Gene Therapy, 26, 593-602. http://dx.doi.org/10.1089/hum.2015.035

[45] Evans, C.H., Ghivizzani, S.C. and Robbins, P.D. (2013) Arthritis Gene Therapy and Its Tortuous Path into the Clinic. Translational Research, 161, 205-216. http://dx.doi.org/10.1016/j.trsl.2013.01.002

[46] Ishihara, A., Bartlett, J.S. and Bertone, A.L. (2012) Inflammation and Immune Response of Intra-Articular Serotype 2 Adeno-Associated Virus or Adenovirus Vectors in a Large Animal Model. Arthritis, 2012, Article ID: 735472 http://dx.doi.org/10.1155/2012/735472

[47] Onuora, S. (2014) Osteoarthritis, Targeting Rac1 via Microparticle-Based Drug Delivery System Protects OA Cartilage in Vivo. Nature Reviews Rheumatology, 10, 1. http://dx.doi.org/10.1038/nrrheum.2013.192

[48] Brama, P.A., Firth, E.C., van Weeren, P.R., Tuukkanen, J., Holopainen, J., Helminen, H.J., et al. (2009) Influence of Intensity and Changes of Physical Activity on Bone Mineral Density of Immature Equine Subchondral Bone. Equine Veterinary Journal, 41, 564-571. http://dx.doi.org/10.2746/042516409X429437

[49] Mengshol, J.A., Vincenti, M.P., Coon, C.I., Barchowsky, A. and Brinckerhoff, C.E. (2000) Interleukin-1 Induction of Collagenase 3 (Matrix Metalloproteinase 13) Gene Expression in Chondrocytes Requires p38, c-Jun N-Terminal Kinase, and Nuclear Factor KappaB, Differential Regulation of Collagenase 1 and Collagenase 3. Arthritis and Rheumatism, 43, 801-811. http://dx.doi.org/10.1002/1529-0131(200004)43:4<801::AID-ANR10>3.0.CO;2-4

[50] Meszaros, E. and Malemud, C.J. (2012) Prospects for Treating Osteoarthritis, Enzyme-Protein Interactions Regulating Matrix Metalloproteinase Activity. Therapeutic Advances in Chronic Disease, 3, 219-229. http://dx.doi.org/10.1177/2040622312454157

[51] Raina, A., Kumar, S., Shrivastava, R. and Mitra, A. (2015) Testis Mediated Gene Transfer, in Vitro Transfection in Goat Testis by Electroporation. Gene, 554, 96-100. http://dx.doi.org/10.1016/j.gene.2014.10.030

[52] Zhang, X., Mao, Z. and Yu, C. (2004) Suppression of Early Experimental Osteoarthritis by Gene Transfer of Interleukin-1 Receptor Antagonist and Interleukin-10. Journal of Orthopaedic Research, 22, 742-750. http://dx.doi.org/10.1016/j.orthres.2003.12.007 